\title{
Tracking the movement of surgical tools in a virtual temporal bone dissection simulator
}

\author{
Marco Agus ${ }^{1}$, Andrea Giachetti ${ }^{1}$, Enrico Gobbetti ${ }^{1}$, Gianluigi Zanetti ${ }^{1}$, and \\ Antonio Zorcolo ${ }^{1}$ \\ CRS4, VI Strada Ovest, Z. I. Macchiareddu, I-09010 Uta (CA), Italy \\ \{magus, giach, gobbetti, zag, zarco\}@crs4.it, \\ WWW home page:http://www.crs4.it
}

\begin{abstract}
In this paper we present the current state of our research on simulation of temporal bone surgical procedures. We describe the results of tests performed on a virtual surgical training system for middle ear surgery. The work is aimed to demonstrate how expert surgeons and trainees can effectively use the system for training and assessment purposes. Preliminary kinematic and dynamic analysis of simulated mastoidectomy sessions are presented. The simulation system used is characterized by a haptic component exploiting a bone-burr contact and erosion simulation model, a direct volume rendering module as well as a timecritical particle system to simulate secondary visual effects, such as bone debris accumulation, blooding, irrigation, and suction.
\end{abstract}

\section{Introduction}

Temporal bone drilling is an extremely delicate task common to several surgical procedures. A successful execution of temporal bone dissection requires a high level of dexterity, experience, and knowledge of the patient anatomy. The current primary teaching tool to acquire these skills is dissection of human cadavers. The physical limitations and decreased availability of the material - as well as its high handling and disposal cost and the risks associated to transmission of diseases - are, however, making this training method increasingly problematic. A VR simulator realistically mimicking a patient-specific operating environment would, therefore, significantly contribute to the improvement of surgical training in this context.

A number of groups are developing virtual reality surgical simulators for bone dissection. Early systems (e.g. [1]) focused on increasing the understanding of the anatomy by providing specialized visualization tools of static models, while following projects such as the VrTool [2] and the VOXEL-MAN system [3, 4] mainly concentrate on the accurate visual presentation of free-form volumesculpting operations. Others systems, such as the Ohio Virtual Temporal Bone Dissection simulator [5-7] and IERAPSI simulator [8,9] aim instead at realistically mimicking the visual and haptics effects of a real operation. The IERAPSI system is a visual and haptic surgical simulator, characterized by a physically 
based contact model, the use of patient specific data, and the focus on validating the haptic model with experimental data. References $[8,9]$ provide a general overview of the project, mostly covering pre-operative planning; reference [10] focuses on the human factor analysis; while reference [11] presents an implementation of visual and haptic simulation of bone dissection based on a "first principles" model. The visual and haptic simulation is based upon the use of patient specific digital data acquired from $\mathrm{CT}$ scanners and 3D volume models representing the different materials around the temporal bone. It involves also a physical model of the bone-burr interaction that provides impulses to haptic devices(Sensable's Phantoms), a direct volume rendering component and other physically based visual effects. The simulator has been completed and is currently being tested by experienced surgeons and trainees. A complete description of the system architecture and of the algorithms implemented can be found in $[11,12]$.

In this paper, we present the current state of our research on simulation of temporal bone surgical procedures. We report the preliminary results of tests performed on our virtual surgical training system. The data acquisition and analysis involves all the bone-burr interaction dynamic parameters in a series of simulated specific interventions performed by trainees and experienced surgeons. The specialty considered in these sessions is the basic mastoidectomy, that represents the most superficial and common surgery of the temporal bone, and it is undertaken by a wide range of surgeons in everyday practice. The procedure consists in the removal of the air cavities just under the skin behind the ear itself, and it is performed for chronic infection of the mastoid air cells (mastoiditis).

The rest of the paper is organized as follows. Section 2 provides a short description of the virtual surgical training system, while section 3 illustrates our preliminary results with regards to the surgical simulator testing as well as the kinematic and dynamic analysis of the basic mastoidectomy phases.

\section{Methods and tools}

Our surgical simulator has been designed following the requirements identified in a human factor analysis [8,9]. The analysis involved a review of existing documentation, training aids, and video recordings, interviews with experienced operators, as well as direct observation of the procedure being performed in theater. The results of our analysis show that the simulator must include burr-bone contact detection, bone erosion, generation of haptic response, and synthesis of secondary visual effects, such as bone debris accumulation, blooding, irrigation, and suction [10]. The human perceptual requirements of a simulator impose very stringent constraints on performance, making bone dissection simulation a technological challenging task.

We harnessed the difference in complexity and frequency requirements of the visual and haptic simulations by modeling the system as a collection of loosely coupled concurrent components. The haptic component exploits a multiresolution representation of the first two moments of the bone density to rapidly compute contact forces and determine bone erosion. 
The force estimation is based on a physically based contact and erosion model loosely based on Hertz contact theory. The actual bone erosion is implemented by decreasing the density of the voxels that are in contact with the burr in a manner that is consistent with the predicted local mass flows. The method complexity scales, however, with the cube of the burr tip radius, imposing important limitations on the surgical tool size. A thorough description of the method can be found in [12]

The visual component uses a time-critical particle system evolution method to simulate secondary visual effects, such as bone debris accumulation, blooding, irrigation, and suction. The system runs on two interconnected multiprocessor machines. The data is initially replicated on the two machines. The first is dedicated to the high-frequency tasks: haptic device handling and bone removal simulation, which run at $1 \mathrm{KHz}$. The second concurrently runs, at about $15-20 \mathrm{~Hz}$, the low-frequency tasks: bone removal, fluid evolution and visual feedback. The
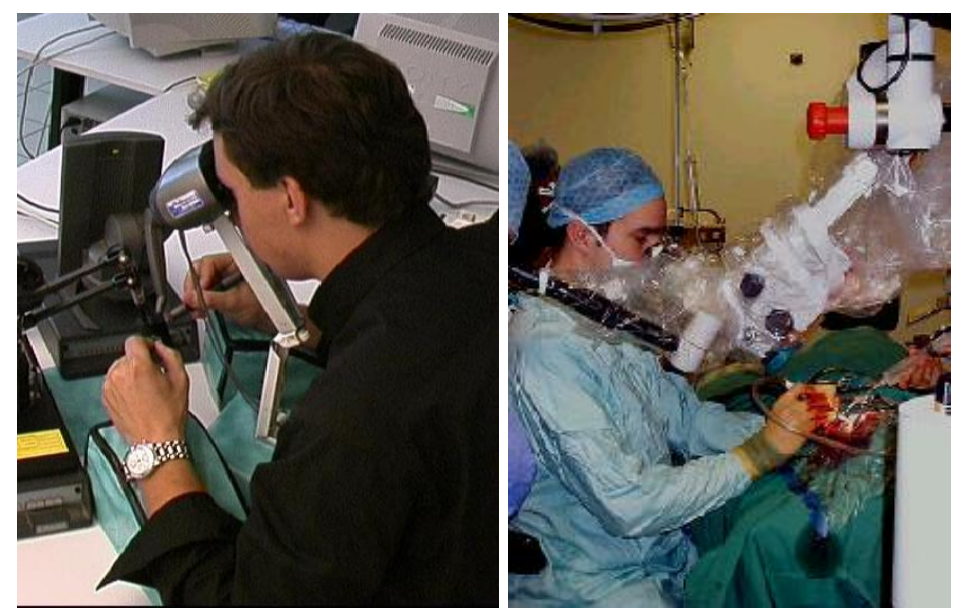

Fig. 1. Surgical simulator setup vs operating room: on the left the virtual surgical training system user interface is composed by two phantom devices that provide force feedback for sucker and burr, as well as an $\mathrm{N}$-vision binocular display that presents images to the user; it simulates the real feelings of the surgeon in the operating room(right)

two machines are synchronized using one-way message passing via the Stanford VRPN library[13]. The Virtual-Reality Peripheral Network (VRPN) system provides a device-independent and network-transparent interface to virtual-reality peripherals. This communication library provides also a suitable mean to record complete traces of the training sessions, which can then be processed off-line by data analysis tools. 


\section{Results}

Our current training system is configured as follows: a single-processor PIV/1500 $\mathrm{MHz}$ with $256 \mathrm{MB}$ PC133 RAM for the high-frequency tasks (haptics loop $(1 \mathrm{KHz})$ and interprocess communication loop); a dual-processor PIII/800 MHz with 512 MB PC800 RAM and a NVIDIA GeForce 4 Ti 4600 and running a 2.4 linux kernel, for the low frequency tasks( receiving loop, simulator evolution and visual rendering); a Phantom Desktop and a Phantom 1.0 haptic devices, that provide $6 \mathrm{DOF}$ tracking and 3DOF force feedback for the burr/irrigator and the sucker; a n-vision VB30 binocular display for presenting images to the user.The performance of the prototype is sufficient to meet timing constraints for display and force-feedback, even though the computational and visualization platform is constructed from affordable and widely accessible components.We are currently using a volume of $256 \times 256 \times 128$ cubical voxels ( $0.3 \mathrm{~mm}$ side) to represent the region where the operation takes place.

We are extensively testing the virtual surgical training system in collaboration with surgeons of the Department of NeuroScience of the University of Pisa. In particular, contact model parameters and erosion factors have been tuned according to their indications and there is consensus that they represent a good approximation of reality. Using the tuned system, surgeons can perform complete virtual surgery procedures with satisfactory realism. The possibility of recording dynamic values of a surgical training session provides new opportunities for the analysis and the evaluation of procedures. Different surgical procedure could be recognized by the system and it becomes possible to use the recorded values also to compare the behavior of expert surgeons and trainees in order to evaluate surgical skills.Current available data show consistency between different training sessions of the same user. Average forces exerted by burr are between 0.7 and $1.3 \mathrm{~N}$ for the expert surgeon and between 0.8 and $1.1 \mathrm{~N}$ for trainees, while average tool velocities are between 8.0 and $12.0 \mathrm{~m} / \mathrm{sec}$ for the expert surgeon and 10.0 and $17.0 \mathrm{~m} / \mathrm{sec}$ for trainees.In order to evaluate the possibility of characterizing different procedures according to dynamical parameters computed by the simulator, we recorded all the parameters (i.e. burr and sucker positions and velocities, force vectors, voxels removed) during a series of simulated mastoidectomy procedures. We analyzed four steps of the mastoidectomy procedure. In the first, the surgeon removes the cortex. The drill is applied to the mastoid cortex immediately posterior to the spine of Henle and draws two perpendicular cuts, the first along the temporal line and the second toward the mastoid tip. Then the mastoid cortex is then removed in a systematic fashion of saucerization.

Figure2A shows a snapshot of the scene viewed by the trainee during this step and on the right plots of the force module and of the material removed as a function of time. The second step is the cavity saucerization: before a deeper penetration in the antrum, it is necessary to perform a wide cortical removal and the posterior canal should be thinned so that the shadow of an instrument can be seen through the bone when the canal skin is elevated. Snapshot and

plots relative to this step are shown in Figure2B. In the next phase considered there is the identification of the mastoid antrum. It can be identified as a larger 

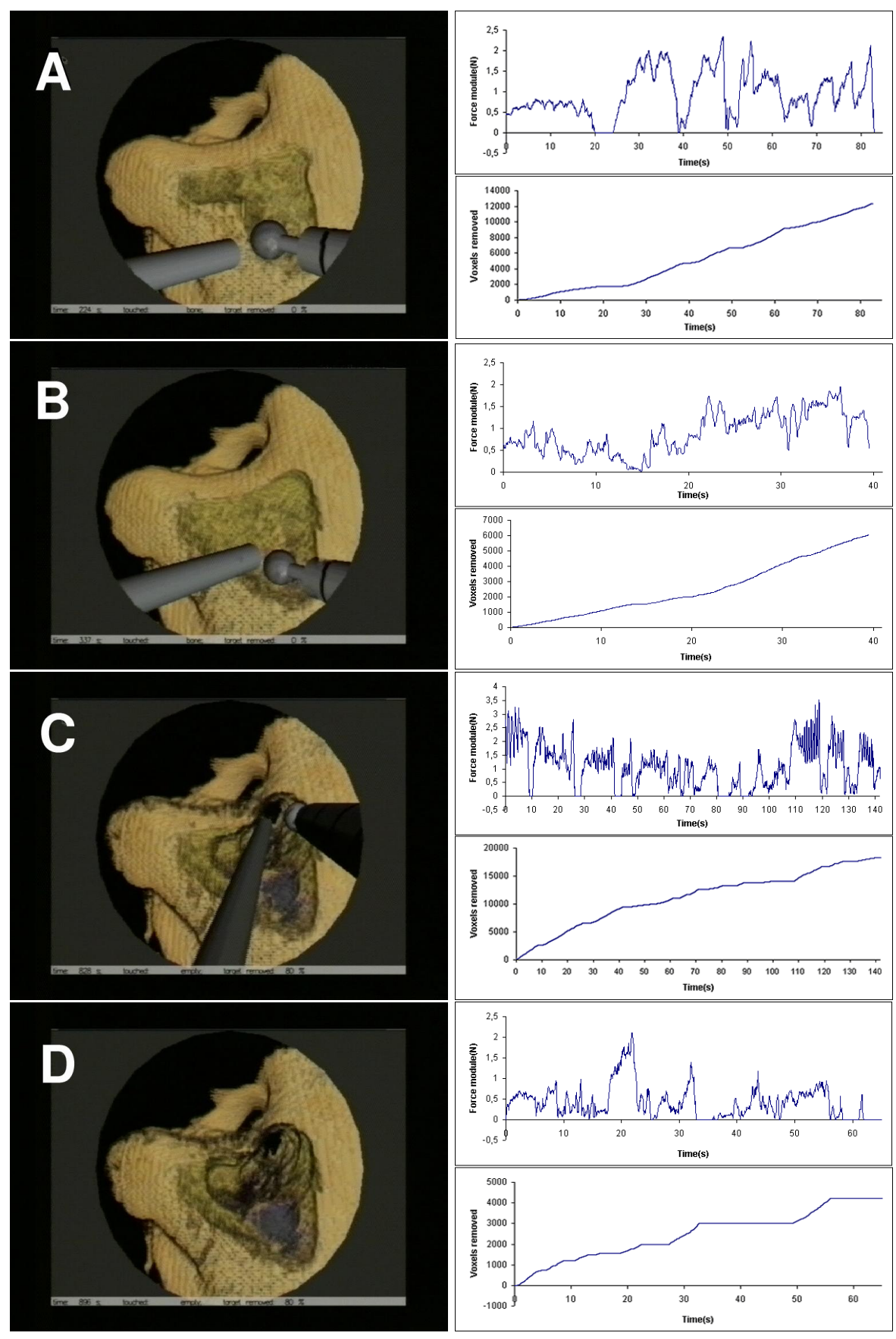

Fig. 2. Snapshot of the simulator (left) and plots of the force modulus and of the bone removal vs time (right) for the four masoidectomy phases considered:A: cortex removal, B: cavity saucerization, C: identification of the mastoid antrum, D: localiztion of the facial recess 
air-containing space at whose bottom lies the basic landmark of the smoothly contoured, hard, labyrinthine bone of the horizontal semicircular canal. The localization of this canal allows exposure of the fossa incudis, the epitymphanum anteriorly and superiorly and the external genu of the facial nerve medially and inferiorly. Snapshot and plots relative to this step are shown in Figure2C. The final part of the basic mastoidectomy is represented in Figure2D. During this step several landmarks are identified, and also the facial recess area is discovered. Force and voxel removal plots show that each step in the surgical procedure can be characterized by different actions. In the first step, the force plot presents evident peaks and valleys due to the necessity of creating holes to start the bone removal. In the second step the force is more continuous and not too high. During the mastoid antrum exposure the force is irregular and reaches higher values, up to $3 \mathrm{~N}$. The removal rate is similar, about 10.000 voxel removed per second. Finally the last considered phase is characterized by large pauses where there is no voxel removal and even when removal is present its rate is lower than in the previous steps, indicating that critical sites have been reached and consequently burring movements are more careful and accurate.

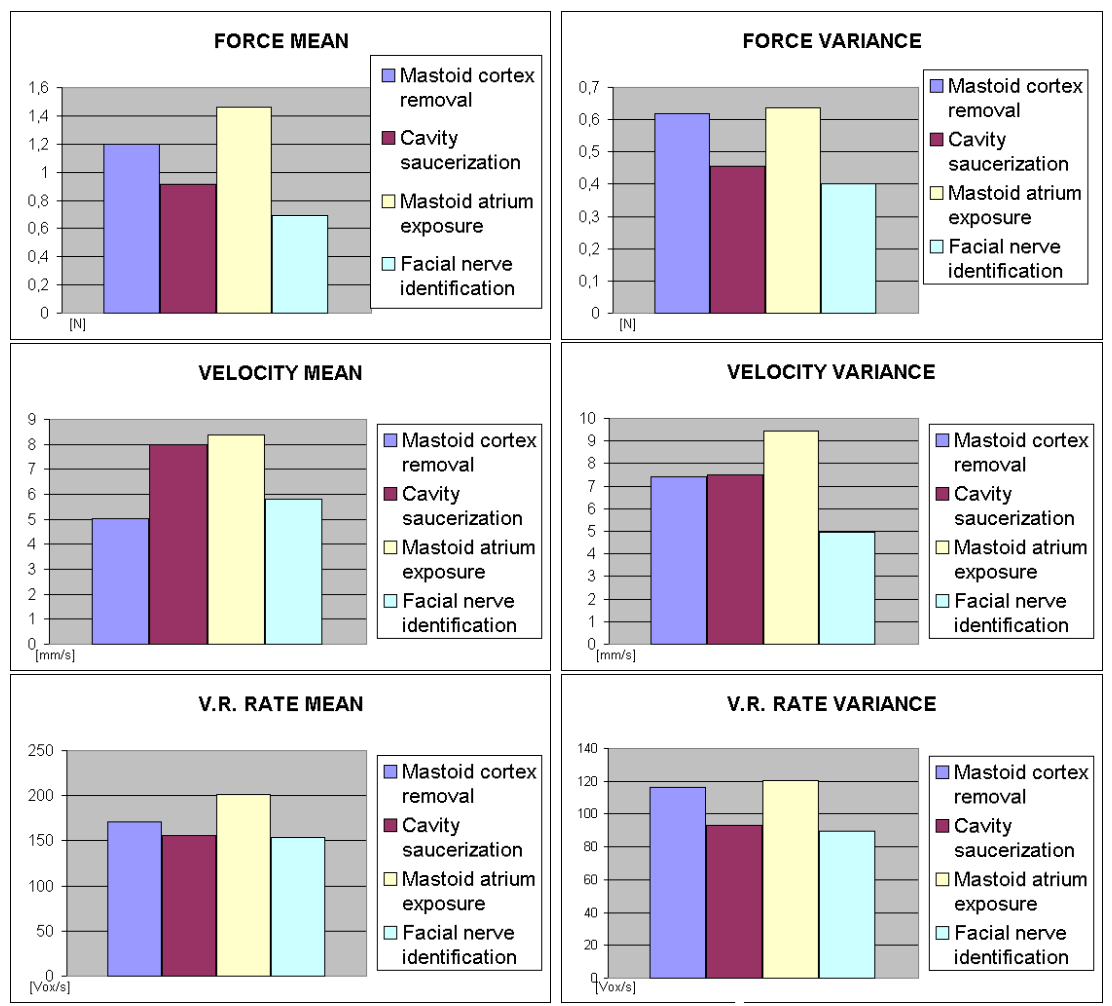

Fig. 3. Average value and variance of the force modulus, velocity and bone voxels removed during the four mastoidectomy phases considered 
These facts can be pointed out just taking statistical values relative to the considered steps displayed in figures 3. It is possible, for example, to distinguish two phases with high average values of force and bone removal and two with lower values. The two phases with high bone removal can be distinguished by the average burr velocity: in the mastoid cortex removal, where the user try to start new paths for the bone removal, the velocity is limited, while in the mastoid atrium exposure, where the user removes small quantities of material burr's movements are much faster. The cavity saucerization and the facial nerve identification phases, characterized by lower force values can also be distinguished by correlating with the burr bit movements speed. In fact, in the first phase the burr moves quickly along already determined paths, while in the second it is moved slowly - and carefully - since there is an high risk of damaging the facial nerve.

\section{Conclusions and Future Work}

This paper was aimed to describe the current state of our research in the field of virtual simulation of temporal bone surgical procedures. We presented preliminary results of the analysis of experimental data acquired during validating session of a novel virtual surgical training system for middle ear surgery. Tests are performed by expert surgeons and trainees and data are acquired in a controlled environment. These data can provide to the surgical community useful information to improve the training methods for critical surgical procedures involving bone dissection. We are currently in the process of acquiring experimental data also to compare the dynamic behavior of real materials, burr tips, and burring velocities with the simulated ones. We are also working on defining metrics appropriate to the quantitative analysis of virtual training session traces.

\section{Acknowledgments}

We would like to thank Pietro Ghironi for the precious technical support, Stefano Sellari Franceschini and the surgical residents Michele D'Anteo and Simone Valori for their performances during the training sessions. We also acknowledge the NIH National Research Resource in Molecular Graphics and Microscopy at the University of North Carolina at Chapel Hill for the VRPN library.

\section{References}

1. T. Harada, S. Ishii, and N. Tayama, "Three-dimensional reconstruction of the temporal bone from histological sections," Arch Otolaryngol Head Neck Surg, vol. 114, pp. 1139-1142, 1988.

2. RB Kuppersmith, R Johnston, D Moreau, RB Loftin, and H Jenkins, "Building a virtual reality temporal bone dissection simulator," in Medicine Meets Virtual Reality 5, J. D. Westwood, Ed., Amsterdam, The Netherlands, January 1997, pp. 180-186, IOS Press. 
3. Bernhard Pflesser, Andreas Petersik, Ulf Tiede, Karl Heinz Hohne, and Rudolf Leuwer, "Volume based planning and rehearsal of surgical interventions," in Computer Assisted Radiology and Surgery, Proc. CARS 2000, Excerpta Medica International Congress, Heinz U. Lemke et al., Ed., Elsevier, Amsterdam, 2000, 1214, pp. 607-612.

4. Bernhard Pflesser, Andreas Petersik, Ulf Tiede, Karl Heinz Hohne, and Rudolf Leuwer, "Haptic volume interaction with anatomic models at sub-voxel resolution," in 10th International Symposium on Haptic Interfaces for Virtual Environment and Teleoperator Systems,Proc. Haptics 2002, 2002, pp. 66-72.

5. G. Wiet, J. Bryan, D. Sessanna, D. Streadney, P. Schmalbrock, and B. Welling, "Virtual temporal bone dissection simulation," in Medicine Meets Virtual Reality 2000, J. D. Westwood, Ed., Amsterdam, The Netherlands, January 2000, pp. 378384, IOS Press.

6. Jason Bryan, Don Stredney, Greg Wiet, and Dennis Sessanna, "Virtual temporal bone dissection: A case study," in IEEE Visualization, 2001, pp. 497-500.

7. D. Stredney, G. Wiet, J. Bryan, D. Sessanna, J. Murakami, O. Schamllbrock, K. Powell, and B. Welling, "Temporal bone dissection simulation - an update," in Medicine Meets Virtual Reality 2002, J. D. Westwood, H. M. Hoffmann, G. T. Mogel, and D. Stredney, Eds. Jan. 2002, pp. 507-513, IOS Press.

8. N. W. John, N. Thacker, M. Pokric, A. Jackson, G. Zanetti, E. Gobbetti, A. Giachetti, R. J. Stone, J. Campos, A. Emmen, A. Schwerdtner, E. Neri, S. Sellari Franceschini, and F. Rubio, "An integrated simulator for surgery of the petrous bone," in Medicine Meets Virtual Reality 2001, J. D. Westwood, Ed., Amsterdam, The Netherlands, January 2001, pp. 218-224, IOS Press.

9. Marco Agus, Andrea Giachetti, Enrico Gobbetti, Gianluigi Zanetti, and Antonio Zorcolo, "A multiprocessor decoupled system for the simulation of temporal bone surgery," Computing and Visualization in Science, vol. 5, no. 1, 2002.

10. Marco Agus, Andrea Giachetti, Enrico Gobbetti, Gianluigi Zanetti, Nigel W. John, and Robert J. Stone, "Mastoidectomy simulation with combined visual and haptic feedback," in Medicine Meets Virtual Reality 2002, J. D. Westwood, H. M. Hoffmann, G. T. Mogel, and D. Stredney, Eds. Jan. 2002, pp. 17-23, IOS Press.

11. Marco Agus, Andrea Giachetti, Enrico Gobbetti, Gianluigi Zanetti, and Antonio Zorcolo, "Real-time haptic and visual simulation of bone dissection," in Proc. of Ieee Virtual Reality 2002, Orlando, FL, USA, March 2002, pp. 209-216.

12. Marco Agus, Andrea Giachetti, Enrico Gobbetti, Gianluigi Zanetti, and Antonio Zorcolo, "Adaptive techniques for real-time haptic and visual simulation of bone dissection," in Proc.of IEEE Virtual Reality 2003, 2003.

13. Il Russell M. Taylor, Thomas C. Hudson, Adam Seeger, Hans Weber, Jeffrey Juliano, and Aron T. Helser, "VRPN: a device-independent, network-transparent vr peripheral system," in Proceedings of the ACM symposium on Virtual reality software and technology. 2001, pp. 55-61, ACM Press. 